\title{
ON THE INTEGRATION OF DIFFUSION EQUATIONS IN RIEMANNIAN SPACES
}

\author{
KôSAKU YOSIDA
}

1. Introduction. Let $R$ be a connected domain of an infinitely differentiable, orientable, $m$-dimensional $(m \geqq 2)$ Riemannian space with the metric $d s^{2}=g_{i j}(x) d x^{i} d x^{j}$. Under a certain "continuity condition" of Lindeberg's type, the temporally homogeneous stochastic process in $R$ is governed by a pair of equations: ${ }^{1}$

$$
\begin{array}{rlrl}
\frac{\partial f(x, t)}{\partial t}= & b^{i j}(x) \frac{\partial^{2} f(x, t)}{\partial x^{i} \partial x^{i}}+a^{i}(x) \frac{\partial f(x, t)}{\partial x^{i}}, & & t \geqq 0, \\
\frac{\partial h(x, t)}{\partial t}= & \frac{1}{(g(x))^{1 / 2}} \frac{\partial^{2}}{\partial x^{i} \partial x^{i}}\left((g(x))^{1 / 2} b^{i j}(x) h(x, t)\right) & \\
& -\frac{1}{(g(x))^{1 / 2}} \frac{\partial}{\partial x^{i}}\left((g(x))^{1 / 2} a^{i}(x) h(x, t)\right), & t \geqq 0 .
\end{array}
$$

These are called the "backward diffusion equation" and the "forward diffusion equation" respectively, the latter being sometimes called the Fokker-Planck's equation. In these equations, the symmetric contravariant tensor $b^{i j}(x)$ is assumed to be such that the quadratic form $b^{i j}(x) \xi_{i} \xi_{j}$ is, for $\sum_{i} \xi_{i}^{2}>0$, greater than 0 in $R$ and $a^{i}(x)$ is assumed to obey, in the coordinate change $x \rightarrow \bar{x}$, the transformation rule

$$
\bar{a}^{i}(\bar{x})=\frac{\partial \bar{x}^{i}}{\partial x^{k}} a^{k}(x)+\frac{\partial^{2} \bar{x}^{i}}{\partial x^{k} \partial x^{s}} b^{k s}(x) .
$$

Hence the two elliptic differential operators on the right-hand sides of (1.1) and (1.2) are formally adjoint to each other and they have a meaning independent of the local coordinates $\left(x^{1}, \cdots, x^{m}\right)$. We assume that the coefficients $g_{i j}(x), a^{i}(x)$, and $b^{i j}(x)$ are infinitely differentiable functions of the local coordinates $\left(x^{1}, \cdots, x^{m}\right)$.

The purpose of the present note is to prove the following three theorems.

Theorem 1. Let $R$ be a compact Riemannian space. Then, to any function $f(x)$, infinitely differentiable in $R$, there corresponds a uniquely determined solution $f(x, t)$ of (1.1) satisfying the conditions:

Received by the editors March 28, 1952.

1 A. Kolmogoroff, Zur Theorie der stetigen zufälligen Prozesse, Math. Ann. vol. 108 (1933) pp. 149-160. W. Feller, Zur Theorie der stochastischen Prozesse, Math. Ann. vol. 113 (1937) pp. 113-160. 


$$
\begin{aligned}
\lim _{t \downarrow 0} f(x, t) & =f(x) \text { uniformly in } x, \\
\min _{x} f(x) & \leqq f(x, t) \leqq \max _{x} f(x), \text { and } \\
\max _{x} f(x, t) & =\max _{x} f(x) \text { when } f(x) \text { is non-negative. }
\end{aligned}
$$

Theorem 2. Let $R$ be a compact Riemannian space. Then, to any function $h(x)$, infinitely differentiable in $R$, there corresponds a uniquely determined solution $h(x, t)$ of (1.2) satisfying the conditions:

(1.6) $\lim _{t \downarrow 0} \int_{R}|h(x, t)-h(x)| d x=0$, where $d x=(g(x))^{1 / 2} d x^{1} \cdots d x^{m}$, $g(x)=\operatorname{det}\left(g_{i j}(x)\right)$,

(1.7) $\int_{R}|h(x, t)| d x \leqq \int_{R}|h(x)| d x$

and $h(x, t)$ is non-negative with $\int_{R} h(x, t) d x=\int_{R} h(x) d x$ when $h(x)$ is non-negative.

If $R$ is a connected domain with the smooth boundary $\partial R$, Theorem 2 is extended to the following theorem.

THEOREM $2^{\prime}$. Let $D$ be the totality of infinitely differentiable functions $h(x)$ in $R$ with compact carriers (supports in the terminology of $L$. Schwartz) satisfying the boundary condition on $\partial R$ :

$$
\begin{aligned}
& (g(x))^{1 / 2} b^{i j}(x) \frac{\partial h}{\partial x^{i}} \cos \left(n, x^{j}\right) \\
& \quad+\left(\frac{\partial(g(x))^{1 / 2} b^{i j}(x)}{\partial x^{i}}-(g(x))^{1 / 2} a^{j}(x)\right) \cos \left(n, x^{j}\right) h(x)=0
\end{aligned}
$$

( $n$ denotes the outer normal). Then, to every $h(x) \in D$, there corresponds a uniquely determined solution $h(x, t)$ of (1.2) satisfying the conditions (1.6)-(1.7) if and only if the following hypothesis is satisfied:

THE HYPOTHESIS. Let $\left\{R_{k}\right\}$ be a monotone increasing sequence of connected domains $\subseteq R$ such that the boundary $\partial R_{k}$ tends smoothly, as $k \rightarrow \infty$, to the boundary $\partial R$. Then, for any $m>0$, the equation

$$
b^{i j}(x) \frac{\partial^{2} f(x)}{\partial x^{i} \partial x^{j}}+a^{i}(x) \frac{\partial f(x)}{\partial x^{i}}=m f(x)
$$

does not admit a bounded solution $f(x) \neq 0$ satisfying the boundary condition

$$
\lim _{x \rightarrow \infty} \int_{\partial R_{k}}(g(x))^{1 / 2} b^{i j}(x) h(x) \frac{\partial f}{\partial x^{i}} \cos \left(n, x^{j}\right) d S=0
$$

$$
\text { for all } h(x) \in D \text {, }
$$


$d S$ denoting the hypersurface element of $\partial R_{k}$.

These theorems may be proved by refining, with the aid of parametrix considerations, the operator-theoretical integration of the diffusion equations given in the preceding notes. ${ }^{2}$ It is to be remarked that our construction of the parametrix for the general diffusion equation is carried out by an elementary calculation without appealing to the theory of integral equations nor to the power series expansion. It is an extension of the construction due to S. Minakshisundaram and $\AA$. Pleijel. ${ }^{3}$

2. The construction of the parametrix. Let $e(x)$ be an infinitely differentiable function and let

$$
A=A_{x}=b^{i j}(x) \frac{\partial^{2}}{\partial x^{i} \partial x^{i}}+a^{i}(x) \frac{\partial}{\partial x^{i}}+e(x) .
$$

Let $\Gamma=\Gamma(P, Q)=r(P, Q)^{2}$ be the square of the smallest distance of two points $P$ and $Q$ of $R$ according to the new metric $d r^{2}=b_{i j}(x) d x^{i} d x^{j}$, where $\left(b_{i j}(x)\right)=\left(b^{i j}(x)\right)^{-1}$. We have then the lemma.

LEMMA 1. For any positive integer $k$, we may construct the parametrix

$$
\begin{aligned}
& H_{k}(P, Q, t-\tau) \\
& \quad=(t-\tau)^{-m / 2} \exp \left(-\frac{\Gamma(P, Q)}{4(t-\tau)}\right) \sum_{i=0}^{k} u_{i}(P, Q)(t-\tau)^{i}, t>\tau,
\end{aligned}
$$

such that

(2.3) $u_{i}(P, Q)(i=0,1, \cdots, k)$ are infinitely differentiable in the vicinity of $Q=P$ and $u_{0}(P, P)=1$,

$$
\left(-\frac{\partial}{\partial \tau}-A_{Q}\right) H_{k}(P, Q, t-\tau)
$$

$$
=(t-\tau)^{k-m / 2} \exp \left(-\frac{\Gamma(P, Q)}{4(t-\tau)}\right) c_{k}(P, Q),
$$

where $c_{k}(P, Q)$ is infinitely differentiable in the vicinity of $Q=P$.

2 K. Yosida, Integration of Fokker-Planck's equation in a compact Riemannian space, Arkiv. för Matematik vol. 1 (1949) pp. 71-75 (to be referred to as [I]). K. Yosida, Integration of Fokker-Planck's equation with a boundary condition, Journal of the Mathematical Society of Japan vol. 3 (1951) pp. 69-73 (to be referred to as [II]). $\mathrm{K}$. Yosida, Integrability of the backward diffusion equation in a compact Riemannian space, Nagoya Math. J. vol. 3 (1951) pp. 1-4 (to be referred to as [III]).

Some properties of the eigenfunctions of the Laplace operator on Riemannian manifolds, Canadian Journal of Mathematics vol. 1 (1949) pp. 242-256. 
PROOF. We introduce the normal coordinates $y^{\sigma}$ of $Q=\left(x^{1}, \cdots, x^{m}\right)$ in the vicinity of $P$ :

$$
y^{\sigma}=(\Gamma(P, Q))^{1 / 2}\left(\frac{d x^{\sigma}}{d r}\right)_{Q=P} .
$$

Let

$$
d r^{2}=\beta_{i j}(y) d y^{i} d y^{j} .
$$

We have the well known formulae

$$
\Gamma(P, Q)=\beta_{i j}(0) y^{i} y^{i}, \quad \beta_{i j}(y) y^{i}=\beta_{i j}(0) y^{i} .
$$

By virtue of (2.7), the operator

$$
\begin{aligned}
& A=A_{y}=\beta^{i j}(y) \frac{\partial^{2}}{\partial y^{i} \partial y^{i}}+\alpha^{i}(y) \frac{\partial}{\partial y^{i}}+e(y), \\
&\left(\beta^{i j}(y)\right)=\left(\beta_{i j}(y)\right)^{-1},
\end{aligned}
$$

when applied to the function $f(\Gamma, y)$, where $\Gamma$ is considered as a function of $y$, may be written as follows:

$$
A_{y} f=4 \Gamma \frac{\partial^{2} f}{\partial \Gamma^{2}}+4 y^{\sigma} \frac{\partial^{2} f}{\partial \Gamma \partial y^{\sigma}}+M \frac{\partial f}{\partial \Gamma}+N(f),
$$

where

$$
\begin{aligned}
M & =\beta^{i j} \frac{\partial^{2} \Gamma}{\partial y^{i} \partial y^{i}}+\alpha^{i} \frac{\partial \Gamma}{\partial y^{i}}=2 m+O(y), \\
N(f) & =\beta^{i j} \frac{\partial^{2} f}{\partial y^{i} \partial y^{i}}+\alpha^{i} \frac{\partial f}{\partial y^{i}}+e f .
\end{aligned}
$$

The differentiations in $A_{y} f$ and $N(f)$ are to be performed as if $\Gamma$ and $y$ are independent variables. Hence we have

$$
\begin{aligned}
-A_{y} H_{k}= & \sum_{i=0}^{k}-\frac{\Gamma}{4}(t-\tau)^{i-2-m / 2} \exp \left(-\frac{\Gamma}{4(t-\tau)}\right) \\
& +\sum_{i=0}^{k}(t-\tau)^{i-1-m / 2} \exp \left(-\frac{\Gamma}{4(t-\tau)}\right)\left\{y^{\sigma} \frac{\partial u_{i}}{\partial y^{\sigma}}\right. \\
& \left.\quad+\frac{M}{4} u_{i}-N\left(u_{i-1}\right)\right\} \\
& +(t-\tau)^{k-m / 2} \exp \left(-\frac{\Gamma}{4(t-\tau)}\right) N\left(u_{k}\right),
\end{aligned}
$$


where $u_{-1} \equiv 0$ and hence $N\left(u_{-1}\right) \equiv 0$. Therefore, since

$$
\begin{aligned}
&-\frac{\partial}{\partial \tau} H_{k}=\sum_{i=0}^{k}(t-\tau)^{i-1-m / 2} \exp \left(-\frac{\Gamma}{4(t-\tau)}\right) \\
& \cdot u_{i}\left(\frac{-m}{2}+i+\frac{\Gamma}{4(t-\tau)}\right),
\end{aligned}
$$

we obtain Lemma 1 if the $u_{i}$ are successively so chosen that

$$
y^{\sigma} \frac{\partial u_{i}}{\partial y^{\sigma}}+\left(\frac{-m}{2}+i+\frac{M}{4}\right) u_{i}=N\left(u_{i-1}\right),
$$

$u_{i}(P, Q)$ being infinitely differentiable in the vicinity of $Q=P$ with $u_{-1} \equiv 0$ and $u_{0}(P, P)=1$. Such $u_{i}$ may be determined, in view of the order relation

$$
M=2 m+O(y) .
$$

For this purpose, put $y^{\sigma}=\eta^{\sigma} s$ and transform (2.10) into ordinary differential equations in $s$ containing the parameters $\eta$. The equations are integrated by

$$
\begin{aligned}
& u_{0}(P, Q)=\exp \left(-\int_{0}^{8} s^{-1}\left(\frac{-m}{2}+\frac{M}{4}\right) d s\right. \\
& u_{i}(P, Q)=u_{0} s^{-i} \int_{0}^{8} s^{i-1} u_{0}^{-1} N\left(u_{i-1}\right) d s \quad(i=1, \cdots, k) .
\end{aligned}
$$

REMARK. By (2.9) and (2.10), we have

$$
\begin{aligned}
A_{Q} u_{0}(P, Q) \Gamma(P, Q)^{(2-m) / 2} & =N\left(u_{0}(P, Q)\right) \Gamma(P, Q)^{(2-m) / 2}, m \geqq 3, \\
A_{Q} u_{0}(P, Q) \log \Gamma(P, Q) & =N\left(u_{0}(P, Q)\right) \log \Gamma(P, Q), \quad m=2 .
\end{aligned}
$$

We have thus obtained the parametrix for the elliptic differential operator $A$.

3. The proof of Theorem $2^{\prime}$. Let $e \equiv 0$ in the operator $A$ and let

$$
\begin{aligned}
\left(A^{\prime} h\right)(x)= & \frac{1}{(g(x))^{1 / 2}} \frac{\partial^{2}}{\partial x^{i} \partial x^{j}}\left((g(x))^{1 / 2} b^{i j}(x) h(x)\right) \\
& +\frac{1}{(g(x))^{1 / 2}} \frac{\partial}{\partial x^{i}}\left(-(g(x))^{1 / 2} a^{i}(x) h(x)\right)
\end{aligned}
$$

be the formally adjoint operator of $A$. Let $L_{1}(R)$ be the Banach space of the totality of the functions $h(x)$ integrable with respect to $d x$ in 
$R$, metrized by the norm $\|h\|=\int_{R}|h(x)| d x . D$ is a dense linear subset of $L_{1}(R)$. We take the operator $A^{\prime}$ to be an additive operator defined on $D \subseteq L_{1}(R)$ into $L_{1}(R)$, and let $\tilde{A}^{\prime}$ be the smallest closed extension of the operator $A^{\prime}$. Then there exists a uniquely determined oneparameter semi-group ${ }^{5}$ of linear operators $T_{t}$ on $L_{1}(R)$ into $L_{1}(R)$ satisfying the conditions

$$
\begin{aligned}
& \left.T_{t} T_{s}=T_{t+s}(t, s) \geqq 0\right), T_{0}=\text { the identity, } \\
& \text { strong } \lim _{t \rightarrow t_{\text {s }}} T_{t} h=T_{t_{0}} h \quad \text { for } h \in L_{1}(R),
\end{aligned}
$$

$\left(T_{t} h\right)(x)$ is non-negative and $\left\|T_{t} h\right\|=\|h\|$ when $h(x)$ is non-negative,

$$
\begin{aligned}
& \partial_{t} T_{t} h=\text { strong } \lim _{\delta \rightarrow 0} \frac{T_{t+\delta} h-T_{t} h}{\delta}=\tilde{A}^{\prime} T_{t} h \text { for } h \text { in the domain } \\
& \tilde{D}^{\prime} \text { of the operator } \tilde{A}^{\prime},
\end{aligned}
$$

if and only if the hypothesis of Theorem $2^{\prime}$ is satisfied.

We thus have to show that the function $h(x, t)=\left(T_{t} h\right)(x)$ is equivalent (in the sense to be explained below) to a function which is continuously differentiable once in $t$ and twice in $x$.

For this purpose we prepare two lemmas.

LEMma 2. Let $f(x, t)$ be an infinitely differentiable function which vanishes outside a compact coordinate neighborhood of $P$. Then

$$
\begin{aligned}
\int_{R} h(y, t) f(y, t) d y & \\
= & \int_{R} h(y, 0) f(y, 0) d y \\
& \quad+\int_{0}^{t} d \tau \int_{R}\left\{\partial_{\tau} h(y, \tau) f(y, \tau)+h(y, \tau) \frac{\partial f(y, \tau)}{\partial \tau}\right\} d y .
\end{aligned}
$$

Proof. By (3.2), $h(y, \tau)$ is strongly differentiable in $\tau$ with the differential quotient $\tilde{A}_{y}^{\prime} h(y, \tau)$. Hence $h(y, \tau) f(y, \tau)$ is weakly differ-

${ }^{4}$ See [II]. Cf. also [I]. The hypothesis is surely satisfied when $R$ is a connected domain in $m$-dimensional Euclidean space whose boundary lies entirely in the compact part of the space and when, moreover, the operator $A$ is the usual Laplacian. See K. Yosida, $A$ theorem of Liouville's type for meson equation, Proc. Imp. Acad. Tokyo vol. 27 (1951) pp. 214-215.

5 E. Hille, Functional analysis and semi-groups, New York, 1948. Cf. also K. Yosida, On the differentiability and the representation of one-parameter semi-group of linear operators, Journal of the Mathematical Society of Japan vol. 1 (1948) pp. 15-21. 
entiable in $\tau$ with the differential quotient

$$
f(y, \tau) \tilde{A}_{y}^{\prime} h(y, \tau)+\frac{\partial f(y, \tau)}{\partial \tau} h(y, \tau)
$$

which is strongly continuous in $\tau$. Thus, by integration, we obtain (3.3).

Lemma 3. We have

$$
\begin{aligned}
\int_{R} h(y, t) f(y, t) d y & =\int_{R} h(y, 0) f(y, 0) d y \\
= & \int_{0}^{t} d \tau \int_{R}\left\{h(y, \tau)\left(\frac{\partial f(y, \tau)}{\partial \tau}+A_{y} f(y, \tau)\right)\right\} d y .
\end{aligned}
$$

Proof. The right-hand side is, by (3.2) and (3.3), equal to

$$
\begin{aligned}
& -\int_{0}^{t} d \tau \int_{R}\left\{h(y, \tau)\left(-\frac{\partial f(y, \tau)}{\partial \tau}-A_{y} f(y, \tau)\right)\right. \\
& \left.=\left[\int_{R} h(y, \tau) f(y, \tau) d y\right]_{0}^{t}-f(y, \tau)\left(\partial_{\tau} h(y, \tau)-\tilde{A}_{y}^{\prime} h(y, \tau)\right)\right\} d y \\
& +\int_{0}^{t} d \tau \int_{R}\left\{h(y, \tau) A_{y} f(y, \tau)-f(y, \tau) \tilde{A}_{y}^{\prime} h(y, \tau)\right\} d y .
\end{aligned}
$$

That the second integral on the right-hand side is equal to zero may be seen by the following argument. Let $\left\{h_{k}(y, \tau)\right\} \subseteq D$ be such that strong $\lim _{k \rightarrow \infty} h_{k}(y, \tau)=h(y, \tau)$, strong $\lim _{k \rightarrow \infty} A_{y}^{\prime} h_{k}(y, \tau)=\tilde{A}_{y}^{\prime} h(y, \tau)$. The existence of such a sequence $\left\{h_{k}(y, \tau)\right\}$ is assured by the fact that $h(y, \tau)=\left(T_{\tau} h\right)(y)$ is in the domain $\tilde{D}^{\prime}$ of the operator $\tilde{A}^{\prime}$ which is the smallest closed extension of the operator $A^{\prime}$ with the domain $D$. We have thus

$$
\begin{aligned}
\int_{R}\left\{h(y, \tau) A_{y} f(y, \tau)\right. & \left.-f(y, \tau) \tilde{A}_{y}^{\prime} h(y, \tau)\right\} d y \\
= & \lim _{k \rightarrow \infty} \int_{R}\left\{h_{k}(y, \tau) A_{y} f(y, \tau)-f(y, \tau) A_{y}^{\prime} h_{k}(y, \tau)\right\} d y .
\end{aligned}
$$

The right-hand integrals are equal to zero as may be seen by Green's integral theorem and the fact that $f(y, \tau)$ vanishes outside a compact coordinate neighborhood of $P$. 
After these preliminaries, we may give:

Proof of the Theorem $2^{\prime}$. We shall apply (3.4) to the case when

$$
f(y, \tau)=f(Q, \tau)=H_{k}(P, Q, t+\epsilon-\tau) \delta(P, Q) \delta\left(P_{0}, P\right) .
$$

Here $P_{0}$ is an arbitrary point of $R$, fixed in the following argument, $\epsilon$ is a positive constant, and

$$
\delta(P, Q)=\alpha(r(P, Q)),
$$

where $\alpha(r)$ denotes an infinitely differentiable function of $r$ satisfying

$$
\alpha(r)=\left(\begin{array}{ll}
1 & \text { for } r \leqq 2^{-1} \eta, \\
\text { between } 0 \text { and } 1 & \text { for } 2^{-1} \eta<r<\eta, \\
0 & \text { for } r \geqq \eta
\end{array}\right.
$$

We assume that the positive constant $\eta$ is chosen so small that

(3.7) the points $Q$ satisfying $\delta\left(P_{0}, P\right) \delta(P, Q)>0$ are contained in a compact coordinate neighborhood of $P_{0}$.

We have thus

$$
\begin{aligned}
& \int_{R} h(Q, t) H_{k}(P, Q, \epsilon) \delta\left(P_{0}, P\right) \delta(P, Q) d y \\
&-\int_{R} h(Q, 0) H_{k}(P, Q, t+\epsilon) \delta\left(P_{0}, P\right) \delta(P, Q) d y \\
&=-\int_{0}^{t} d \tau \int_{R} h(Q, \tau) K_{k}(P, Q, t+\epsilon-\tau) d y
\end{aligned}
$$

where

$$
\begin{aligned}
K_{k}(P, Q, & t+\epsilon-\tau) \\
= & \left(-\frac{\partial}{\partial \tau}-A_{Q}\right)\left(H_{k}(P, Q, t+\epsilon-\tau) \delta\left(P_{0}, P\right) \delta(P, Q)\right)
\end{aligned}
$$

Let $k$ be taken so large that

$$
\frac{-m}{2}+k \geqq 2 \text {. }
$$

Then, in view of Lemma $1, K_{k}(P, Q, t+\epsilon-\tau)$ is, for $r\left(P_{0}, P\right) \leqq 2^{-1} \eta$, devoid of the singularity even if $(t+\epsilon-\tau)=0$. We shall next show that the first term on the left-hand side of (3.8) tends, as $\epsilon \downarrow 0$, strongly to $h(P, t)$ in the vicinity of $P_{0}$. This may be proved as follows. We have, by (3.6) and (3.7), 


$$
\begin{aligned}
& \int_{R} \delta\left(P_{0}, P\right) d P \mid \int_{R} h(Q, t) H_{k}(P, Q, \epsilon) \delta(P, Q) d y \\
& -h(P, t) \int_{R} H_{k}(P, Q, \epsilon) \delta(P, Q) d y \mid \\
& \leqq C \int_{r\left(P_{0}, Q\right) \leqq 2 \eta}\left(\int_{r\left(P_{0}, P\right) \leqq \eta}|h(Q, t)-h(P, t)| d P\right)\left|H_{k}(P, Q, \epsilon)\right| d y \\
& \leqq C_{1} \int \ldots \int\left(\int\left|h\left(z+\epsilon^{1 / 2 \xi}, t\right)-h(z, t)\right| d z\right) \\
& \cdot \exp \left(-\sum_{i}\left(\xi^{i}\right)^{2}\right) d \xi^{1} \cdots d \xi^{m} .
\end{aligned}
$$

Here $\left(z^{1}, \cdots, z^{m}\right)$ and $\left(z^{1}+y^{1}, \cdots, z^{m}+y^{m}\right)$ are the coordinates of the points $P$ and $Q$ respectively in the coordinate neighborhood of $P_{0}$, defined in (3.7), and $C$ and $C_{1}$ denote suitable positive constants. The inner integral on the right-hand side converges, when $\epsilon \downarrow 0$, to zero boundedly by Lebesgue's theorem.

Therefore, there exists a sequence $\left\{\epsilon_{i}\right\}$ with $\epsilon_{i} \downarrow 0$ such that

$$
\begin{aligned}
h(P, t) \lim _{t \rightarrow \infty} \int_{R} H_{k}\left(P, Q, \epsilon_{i}\right) \delta(P, Q) d y \\
=\int_{R} h(Q, 0) H_{k}(P, Q, t) \delta(P, Q) d y \\
-\int_{0}^{t} d \tau \int_{R} h(Q, \tau) K_{k}(P, Q, t-\tau) d y
\end{aligned}
$$

almost everywhere with respect to $P$ in the vicinity of $P_{0}$. Hence, by (3.10)-(3.11), $h(P, t)$ may be considered to be continuously differentiable once in $t>0$ and twice in $P$ in the vicinity of $P_{0}$ if

$$
\begin{aligned}
& \lim _{\epsilon \downarrow 0} \int_{R} H_{k}(P, Q, \epsilon) \delta(P, Q) d y \text { is positive and twice continuously } \\
& \text { differentiable in } P \text { in the vicinity of } P_{0} .
\end{aligned}
$$

The proof of (3.12) may be obtained as follows. We have

$$
\begin{aligned}
\lim _{\bullet \downarrow 0} \int_{R} H_{k}(P, Q, \epsilon) \delta(P, Q) & d y \\
& =\lim _{\bullet \downarrow 0} \int_{r(P, Q) \leqq \zeta} \epsilon^{-m / 2} \exp \left(-\frac{\Gamma(P, Q)}{4 \epsilon}\right) d y
\end{aligned}
$$


for any positive constant $\zeta$. Hence, by putting

$$
d s^{2}=\gamma_{i j}(y) d y^{i} d y^{j}, \quad y^{i}=\epsilon^{1 / 2} \xi^{i},
$$

we obtain, in view of the arbitrariness of $\zeta$,

$$
\lim _{\epsilon \downarrow 0} \int_{R} H_{k}(P, Q, \epsilon) \delta(P, Q) d y
$$

$$
\begin{aligned}
& =\lim _{\epsilon \downarrow 0} \int \cdots \int_{-\zeta \leqq \epsilon^{1 / 2} \xi^{i} \leq \zeta} \exp \left(-\beta_{i j}(0) \xi^{i} \xi^{j}\right)(\gamma(0))^{1 / 2} d \xi^{1} \cdots d \xi^{m} \\
& =\pi^{m / 2}(\gamma(0))^{1 / 2}(\beta(0))^{1 / 2}=\pi^{m / 2}(g(P))^{1 / 2} /(b(P))^{1 / 2}
\end{aligned}
$$

where

$$
g(P)=\operatorname{det}\left(g_{i j}(P)\right) \text { and } b(P)=\operatorname{det}\left(b_{i j}(P)\right) .
$$

4. The proof of Theorem 1. Let $C(R)$ be the Banach space of the totality of continuous functions $f(x)$ in $R$, metrized by the norm $\|f\|$ $=\max _{x}|f(x)| . D$ is a dense linear subset of $C(R)$. Let $e \equiv 0$ in the operator $A$. We consider this $A$ to be an additive operator defined on $D \subseteq C(R)$ into $C(R)$, and define the smallest closed extension $\tilde{A}$ of $A$. Then $^{6}$ there exists a uniquely determined one-parameter semi-group of linear operators $S_{t}$ on $C(R)$ into $C(R)$ satisfying the conditions:

$$
\begin{aligned}
& S_{b} S_{s}=S_{t+8} \quad(t, s \geqq 0), \quad S_{0}=\text { the identity } I, \\
& \text { strong } \lim _{t \rightarrow t_{0}} S_{t} f=S_{t_{0}} f \text { for } f \in C(R), \\
& \left(S_{t} f\right)(x) \text { is non-negative and } \max _{x}\left(S_{t} f\right)(x)=\max _{x} f(x) \text { if } f(x)
\end{aligned}
$$

is non-negative

$\partial S f=$ strong $\lim _{\delta \rightarrow 0} \frac{S_{t+\delta} f-S_{t} f}{\delta}=\tilde{A} S_{t} f$ for $f$ in the domain $\tilde{D}$ of

the operator $A$.

Therefore, as in $\$ 3$, we may prove that $f(x, t)=\left(S_{t} f\right)(x)$ may be considered to be continuously differentiable once in $t$ and twice in $x$.

\section{NAGOYA UNIVERSITY}

- See [III]. 